\title{
RAPID COMMUNICATION \\ A Regression Analysis Study of the Primary Somatosensory Cortex during Pain
}

\author{
Predrag Petrovic,* Karl Magnus Petersson,* Per Hansson,† and Martin Ingvar* \\ *PET, Cognitive Neurophysiology, Department of Clinical Neuroscience, and †Neurogenic Pain Unit, Multidisciplinary Pain Center, \\ and Department of Rehabilitation Medicine, Karolinska Institute, Karolinska Hospital, Sweden
}

Received J uly 16, 2001

Several functional imaging studies of pain, using a number of different experimental paradigms and a variety of reference states, have failed to detect activations in the somatosensory cortices, while other imaging studies of pain have reported significant activations in these regions. The role of the somatosensory areas in pain processing has therefore been debated. In the present study the left hand was immersed in painfully cold water (standard cold pressor test) and in nonpainfully cold water during $2 \mathrm{~min}$, and PETscans were obtained either during the first or the second minute of stimulation. We observed no significant increase of activity in the somatosensory regions when the painful conditions were directly compared with the control conditions. In order to better understand the role of the primary somatosensory cortex (S1) in pain processing we used a regression analysis to study the relation between a ROI (region of interest) in the somatotopic S1-area for the stimulated hand and other regions known to be involved in pain processing. We hypothesized that although no increased activity was observed in the S1 during pain, this region would change its covariation pattern during noxious input as compared to the control stimulation if it is involved in or affected by the processing of pain. In the nonpainful cold conditions widespread regions of the i psilateral and contralateral somatosensory cortex showed a positive covariation with the activity in the S1-ROI. However, during the first and second minute of pain this regression was significantly attenuated. During the second minute of painful stimulation there was a significant positive covariation between the activity in the S1-ROI and the other regions that are known to be involved in pain processing. Importantly, this relation was significantly stronger for the insula and the orbitofrontal cortex bilaterally when compared to the nonpainful state. The results indicate that the S1-cortex may be engaged in or affected by the processing of pain although no differential activity is observed when pain is compared with the reference condition. 2002 Elsevier Science (USA)

Key Words: positron emission tomography; regional cerebral blood flow; lateral pain system; primary somatosensory cortex; regression analysis; pain; cold pressor test.

\section{INTRODUCTION}

It has been discussed whether the somatosensory cortex is necessary for the processing of pain since the beginning of last century when Head and Holmes failed to find any persistent changes of pain perception in patients with cortical lesions (Head and Holmes, 1911). Later neurophysiological and behavioural studies in humans and monkeys have presented data in favour of the hypothesis that the somatosensory regions participate in acute pain processing (Greenspan and Winfield, 1992; Kenshalo et al., 1988; Kenshalo and I sensee, 1983; Kenshalo et al., 1991; Robinson and Burton, 1980). However, the issue of the nature of the involvement of S1 in pain processing has once again become highlighted because of the disparate results reported from functional neuroimaging studies of pain (Bushnell et al., 1999; Ingvar, 1999; Treede et al., 1999). Some studies have reported robust activations in S1 and S2, while other studies have failed to detect significant changes in the somatosensory regions (Bushnell et al., 1999). The inconsistent findings have raised the question whether the $\mathrm{S} 1$ may be exclusively involved only in specific contexts or stimulus types of pain processing (Treede et al., 1999). Another possibility is that the $\mathrm{S} 1$ is involved in pain processing also in the studies where no increased activation is noted in the contrast analysis. Thus, a lack of S1 activation in functional imaging studies may indicate that S1 is not participating in pain processing at all during certain stimuli/contextual conditions, or that S1 is participating in pain processing but its response is obscured in a subtraction analysis. 
There are several reasons why an approximately constant activity across conditions does not necessarily exclude a region from participation in the processing of noxious input. First, a signal increase may not be observed due to a complex response. S1 has been divided into Broddman area (BA) $3 b$ and 1 which receive wide dynamic range (WDR) nociceptive input from ventral posterior nuclei of the thalamus (VP), but also BA3a which receives input from nociceptive specific lamina 1 relay nucleus of the posterior part of the ventral medial thalamus (VM po) (Craig, 1999). In line with the suggestion above S1 may respond with both activity increases (BA3a) and activity decreases (BA3b and BA1) (Tommerdahl et al., 1996). Such a complex response may obscure a net increase of the rCBF response due to smoothing effects and low resolution in a PET image. F urthermore, there may be an increased activity during pain, which is below the threshold for detection in a subtraction analysis. This may occur if the control condition involves activation of the same regions, or if the response due to pain is small or variable (i.e., lack of statistical power). An indication of the former possibility is that cold, which is not perceived as painful, also has a tendency to activate both the S1 and S2 regions (Craig et al., 1996). The activity increase may be small or abolished also due to attentional modulations, e.g., effects of distraction (Bushnell et al., 1999; Ingvar, 1999; Petrovic et al., 2000a). Apart from changes in the net rCBF response, the activity of S1 may also covary differently with other regions involved in pain processing during the painful conditions as compared to the control conditions. A regression approach could be more sensitive than a subtraction analysis in order to test whether S1 is differently involved in pain processing, especially if the control-state also induces increased $\mathrm{rCBF}$ activity in the $\mathrm{Sl}$ or if the rCBF increase is small. Thus, an alternative to the subtraction approach would be to employ a regression analysis and study the differences between the covariation patterns in the different conditions (e.g., psychophysiological interaction; Friston, 1994; Friston et al., 1997) of the involved somatosensory cortex. This statistical approach will analyse how one area (e.g., the voxels in the predefined pain matrix) is regressed on another area (e.g., the S1-ROI) in a specific condition. It will then compare if this regression changes during another condition. These changes in covariation can be attributed to changes of neural systems influencing each other differently in different conditions or being influenced by other factors such as different input to the brain.

In this study we analyse a data set in which a painful tonic cold stimulation of the hand did not evoke any increased activity in the lateral pain system using the subtraction method (see abstract Petrovic et al., 2000b). S1 is an excellent structure for such analysis because we can predict the approximate position, i.e. define it anatomically without using data from the present study. A similar analysis would be hard to perform for the thalamus since the nuclei processing noxious input are small and blood flow data therefore could potentially be confounded by other nuclei involved in different processes. Likewise, it would be hard to perform such analysis in the anterior cingulate cortex (ACC) since it is not possible to predict exectly which part of the ACC should be involved in pain processing and also different aspects of pain seem to be represented in this large cortical region. In accordance with the reasoning above, we regressed the activity of all intracerebral voxels on the activity of a S1-ROI placed in the somatotopic region for the stimulated hand. This ROI will include activity from the BA3a, $\mathrm{BA} 3 \mathrm{~b}$, and 1 . Thus, an observed pattern of covariations between the somatotopic S1 area for the stimulated hand and other regions known to participate in pain processing during painful stimulation but not during the non-painful control condition, would suggest that this region participates in pain processing although no differential effects were observed in the subtraction analysis. Such effects would argue against the hypothesis based on previous subtraction analysis, that S1 is only involved in specific contexts or stimulus types during noxious stimulation.

\section{MATERIALS AND METHODS}

Seven subjects participated in the PET study, which was approved by the local ethics and radiation safety committees. Subjects participated in a pretest, which included the noxious stimulation, approximately 1 week before the PET-session. All subjects tolerated the cold pressor test and were included in the study. Each subject participated in 12 1-min measurements of the regional cerebral blood flow (rCBF) using a 3-D Ecat Exact HR positron emission tomograph (PET; Wienhard et al., 1994) and bolus injections of $500 \mathrm{MBq}$ [ ${ }^{15}$ O]-butanol (Berridge et al., 1990; Ingvar et al., 1994; Wienhard et al., 1994).

Four different conditions were used in the study. In the painful conditions ( $p a$ and pb) the left hand of the subject was immersed in circulating water of $0+<$ $0.5^{\circ} \mathrm{C}$ during $130 \mathrm{~s}$. In one of the two pain conditions the subjects were scanned during the first minute of noxious cold stimulation (pa), and in the other during the second minute of the same stimulation (pb). The control conditions (ca and cb) were similar to the painful conditions but the water was nonpainfully cold $20 \pm$ $0.5^{\circ} \mathrm{C}$. Thus, the subjects were scanned during the first minute (ca) as well as during the second minute (cb) of the 2-min control stimulation. Each of the four conditions (ca, cb, pa, and pb) was repeated three times in randomized order in three blocks (every block included one of the four conditions). The immersion of the hand was simultaneous with the time of the tracer injection in the pa and ca conditions, i.e., approximately $10 \mathrm{~s}$ 
before start of the bolus arrival in the brain. During the $\mathrm{cb}$ and $\mathrm{pb}$ condition the stimulation onset time was 70 $\mathrm{s}$ before the scanning started. Individual plaster head support was constructed to minimize head movements during the PET imaging. The subjects were told to lay still and not move their hand during the stimulation. The subjects' hand was observed intermittently during the scan and no movements were detected. After each scan pain ratings of the average pain perception for the stimulation period were obtained (visual analogue scale (VAS) ranging from 0 (no pain) to 100 (maximally imaginable pain intensity)).

The measurements of the S1-ROI activity were generated from PET images that were realigned, spatially normalized, isotropic Gaussian filtered $(10 \mathrm{~mm}$ FWHM), proportionally scaled to account for global confounders and transferred into the Karolinska computerized brain atlas (Greitz et al., 1991). The filtering was set to only $10-\mathrm{mm}$ FWHM in this step of the analysis in order to reduce potential influencers from the neighboring motor cortex, posterior parietal cortex and other somatotopic areas in the S1. In this study no increased activity was observed in the S1 during the painful stimulation (Petrovic et al., 2000b). However, in a previous study, painful stimulation of the left hand showed a clear activation of the contralateral S1 during pain as compared to the control condition (Petrovic et al., 2000a). The coordinates of this activation were used to position a spherical region of interest (ROI ; 5-mm radius) in the postcentral gyrus as defined in the CBA. The position of the ROI was then transferred to the adjusted images from this study and the activity in the ROI was then extracted for all scans in each of the four conditions for all subjects. The extracted ROI activity was subsequently used for the regression analysis (see below).

The PET images used for the statistical analysis were realigned, spatially normalized, isotropic Gaussian filtered (16-mm FWHM), proportionally scaled to account for global confounders and transformed into the stereotatic MNI-space as defined by the SPM99b template (http:/ / www.fil.ion.ud.ac.uk/ spm). The extracted activity for each condition in the ROI was corrected for condition specific mean and used as a covariate of interest in the SPM99b (http:/ / www.fil.ion.ud.ac.uk/ spm; F riston et al., 1995). The four different conditions were included as confounding covariates in the linear model (Friston et al., 1995). We then studied differences between the observed regressions (i.e., covariations) in the different conditions employing the general linear model in the SPM99b (Friston, 1994, 1995, 1997).

Given our regionally specific hypothesis, the search for regions that were significantly regressed on the S1-ROI activity was only performed in a predefined functional pain network and effects outside this network are not reported (I ngvar, 1999). Regions included in the functional pain network were the primary somatosensory cortex (S1), the somatosensory associa- tion areas (including S2), the posterior insula, mid/ anterior insula, the anterior cingulate cortex (ACC), the orbitofrontal cortex (obfc), the temporopolar cortex, the thalamus, the amygdala and the brainstem, all bilaterally. Since our analyses were restricted to a priori defined regions of interest we used an uncorrected threshold of 0.001 ( $Z=3.09)$, which can be considered as an approximation to a corrected level of 0.05 for the limited volumes searched. Small volume correction was not used in this study since the exact position of the response areas are only generally defined and not defined as precisely located volumes. The S1-ROI was excluded from the search by excluding the most significant voxel in the S1-ROI and any regressions $20 \mathrm{~mm}$ around this point.

\section{RESULTS}

All subjects reported pain during all the noxious stimulations ( $\mathrm{pa}$ and $\mathrm{pb}$; average pain intensity rating $=53.2 ; \mathrm{SD}=18.7 ; 0=$ no pain and $100=$ highest imaginable pain intensity rated after each 2 min stimulation) and none of the subjects reported pain during the cold stimulations (ca and cb). Consistent with previous studies (for a review see Ingvar, 1999) the main effect of pain $((p a+p b)-(c a+c b))$ induced a significant activation bilaterally in the thalamus, the ACC, and contralaterally in the mid/anterior insula. As in several other pain imaging studies (Bushnell et al., 1999) no differential activity increase was observed in the somatosensory cortex in the subtraction analysis neither for the main effect of pain ( $p a+p b)-(c a+$ $\mathrm{cb})$ ) nor for the different pain conditions ( $\mathrm{pa}-\mathrm{ca}$ ) or (pb - cb) (Petrovic et al., 2000b). No statistical trend of increased S1-activity was observed even at an uncorrected threshold at $\mathrm{P}=0.05$. Instead, significantly decreased activity was observed in the ipsilateral S1 as has been observed previously (Peyron et al., 1999). The contrast analyses are not further elaborated in this article (manuscript in preparation).

During the nonpainful cold conditions activity in widespread regions of the ipsilateral and contralateral primary somatosensory cortex regressed significantly on the activity of the S1-ROI (Table 1, Figs. 1A and 1B). The effect was particularly pronounced in the S1 region contralateral to the S1-ROI, i.e., the somatotopic $\mathrm{S} 1$ region for the nonstimulated hand. In addition, we observed negative regressions in the contralateral thalamus and the brainstem. Although these findings were more widespread and the $Z$ values were higher during $\mathrm{cb}$ a formal test did not disclose any significant differences between the regressions in ca and cb.

This pattern of covariation was not observed during the first minute of pain stimulation (pa) and only the activity of the right orbitofrontal cortex regressed significantly on the S1-ROI activity (Table 1, Fig. 1C). Similarly to the pa-condition, no regressions were ob- 
TABLE 1

Regions Showing a Significant Regression on the S1-ROI and Contrast Analysis between the Regressions

\begin{tabular}{|c|c|c|c|c|}
\hline $\begin{array}{l}\text { Regions which are significantly } \\
\text { regressed on the } \\
\text { S1-ROI activity }\end{array}$ & $\begin{array}{l}\quad[X \text { Y Z }] \\
\text { Contralateral to } \\
\text { the stimulation }\end{array}$ & $Z$ value & $\begin{array}{c}{[X \text { Y Z Z }} \\
\text { Ipsilateral to } \\
\text { the stimulation }\end{array}$ & $Z$ value \\
\hline \multicolumn{5}{|l|}{ 1st minute of cold (ca) } \\
\hline \multicolumn{5}{|l|}{ Positive regressions } \\
\hline S1 & {$[16-3262]$} & 3.79 & {$\left[\begin{array}{lll}-50 & -24 & 46\end{array}\right]$} & 3.67 \\
\hline \multicolumn{5}{|l|}{ Negative regressions } \\
\hline Thalamus & {$[8-46]$} & 3.62 & & \\
\hline Brainstem & {$\left[\begin{array}{lll}12 & -32 & -14\end{array}\right]$} & 3.63 & & \\
\hline \multicolumn{5}{|l|}{ 2nd minute of cold (cb) } \\
\hline \multicolumn{5}{|l|}{ Positive regressions } \\
\hline S1 & {$\left[\begin{array}{lll}2 & -20 & 48\end{array}\right]$} & 6.35 & $\begin{array}{l}{\left[\begin{array}{lll}-44 & -1860 & 60 \\
{[-2} & -22 & 74]\end{array}\right.}\end{array}$ & $\begin{array}{l}5.12 \\
4.39\end{array}$ \\
\hline \multicolumn{5}{|l|}{ Negative regressions } \\
\hline $\begin{array}{l}\text { Thalamus } \\
\text { Brainstem }\end{array}$ & $\begin{array}{l}{[10-106]} \\
\text { activated region }\end{array}$ & 4.65 & & \\
\hline \multicolumn{5}{|l|}{ 1st minute of pain (pa) } \\
\hline \multicolumn{5}{|l|}{ Positive regressions } \\
\hline Obfc & {$\left[\begin{array}{lll}12 & 16 & -22\end{array}\right]$} & 3.26 & & \\
\hline \multicolumn{5}{|l|}{ 2nd minute of pain $(\mathrm{pb})$} \\
\hline \multicolumn{5}{|l|}{ Positive regressions } \\
\hline $\begin{array}{l}\text { S2/posterior insula } \\
\text { ACC }\end{array}$ & $\begin{array}{l}{\left[\begin{array}{lll}48 & -34 & 20\end{array}\right]} \\
{\left[\begin{array}{lll}6 & 6 & 36\end{array}\right]}\end{array}$ & $\begin{array}{l}3.99 \\
3.75\end{array}$ & {$\left[\begin{array}{lll}-48 & -36 & 24\end{array}\right]$} & 3.15 \\
\hline Mid-anterior insula & {$\left[\begin{array}{lll}40 & 0 & -6\end{array}\right]$} & 4.75 & {$\left[\begin{array}{lll}-38 & 2 & -4\end{array}\right]$} & 3.96 \\
\hline Obfc/Mid-/anterior insula & {$\left[\begin{array}{lll}20 & 20 & -18\end{array}\right]$} & 3.65 & {$\left[\begin{array}{lll}-22 & 14 & -14\end{array}\right]$} & 4.04 \\
\hline Contrasts between the & {$[X Y Z]$} & & {$[X$ Y Z $]$} & \\
\hline $\begin{array}{l}\text { regression coefficients in the } \\
\text { different conditions }\end{array}$ & $\begin{array}{l}\text { Contralateral to } \\
\text { the stimulation }\end{array}$ & $Z$ value & $\begin{array}{l}\text { I psilateral to } \\
\text { the stimulation }\end{array}$ & $Z$ value \\
\hline \multicolumn{5}{|l|}{$\mathrm{pb}-\mathrm{pa}$} \\
\hline Mid-anterior insula & {$[36-8-10]$} & 3.91 & & \\
\hline $\begin{array}{l}\mathrm{pa}-\mathrm{ca} \\
\text { Brainstem }\end{array}$ & & & {$[-4-18-14]$} & 3.43 \\
\hline \multicolumn{5}{|l|}{$\mathrm{ca}-\mathrm{pa}$} \\
\hline S1 & {$\left[\begin{array}{lll}56 & -10 & 30\end{array}\right]$} & 3.54 & $\begin{array}{l}{\left[\begin{array}{lll}-50 & -18 & 46\end{array}\right]} \\
{\left[\begin{array}{lll}-52 & -32 & 54\end{array}\right]}\end{array}$ & $\begin{array}{l}3.59 \\
3.41\end{array}$ \\
\hline \multicolumn{5}{|l|}{$\mathrm{pb}-\mathrm{cb}$} \\
\hline Midanterior insula & $\begin{array}{l}{\left[\begin{array}{lll}42 & -12 & 4\end{array}\right]} \\
{[38-4-6]}\end{array}$ & $\begin{array}{l}4.5 \\
4.3\end{array}$ & $-360-4$ & 3.57 \\
\hline Obfc/Mid-/anterior insula & {$[2220-16]$} & 3.89 & {$\left[\begin{array}{lll}-22 & 14 & -14\end{array}\right]$} & 4.05 \\
\hline Brainstem & {$[10-20-6]$} & 3.98 & & \\
\hline \multicolumn{5}{|l|}{$c b-p b$} \\
\hline S1 & {$\left[\begin{array}{lll}0 & -22 & 56\end{array}\right]$} & 3.59 & {$\left[\begin{array}{lll}-50 & -16 & 60\end{array}\right]$} & 3.96 \\
\hline
\end{tabular}

Note SMC, primary sensory motor cortex; Obfc, orbitofrontal cortex.

served between the S1-ROI and other regions of the somatosensory cortex during the second minute of pain (pb; Fig. 1D). However, the activity in other regions of the pain matrix, i.e., the S2/posterior insula, the mid/ anterior insula, the ACC and the orbitofrontal cortex bilaterally now regressed significantly on the S1-ROI activity (Table 1; Figs. 2A-2D). The contrast analysis between these two regressions disclosed that only the activity in the contralateral mid-anterior insula was significantly more positively regressed on the S1-ROI activity during pb as compared to pa. No other differences were discl osed between the regressions in pa and pb. It should also be noted that similar but subsignifi- cant regressions with regions in the pain matrix were observed during pa as during pb. Also, no significant effects were observed in the interaction contrasts of the different regressions of $[(p b-c b)-(p a-c a)]$ or $[(p a-$ ca) $-(\mathrm{pb}-\mathrm{cb})]$.

A formal test of the difference between the regression coefficients of the first minute of cold and the first minute of pain (ca - pa) disclosed that the activity in several S1 regions covaried significantly more positively on the activity of the S1-ROI during ca compared to pa (Table 1). Conversely, the activity of a region in the brainstem covaried significantly more positively with the S1-ROI activity in the comparison pa - ca. 

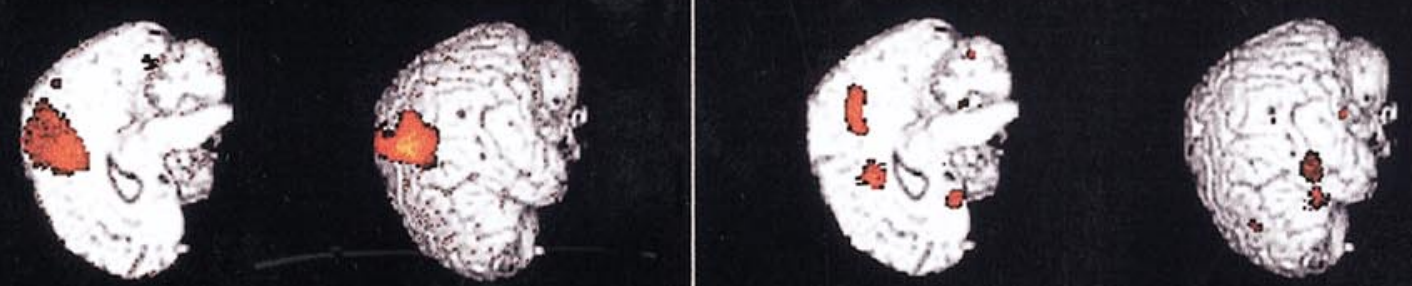

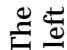
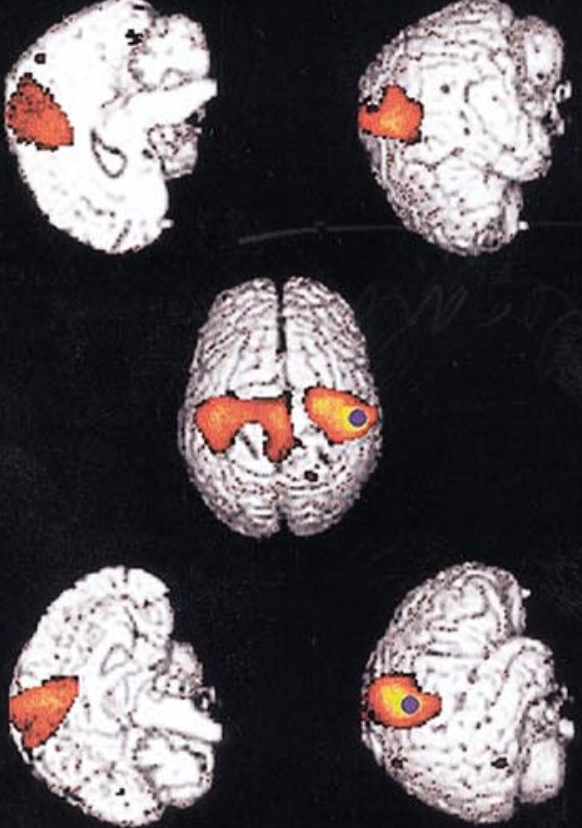

$\oplus$

อ
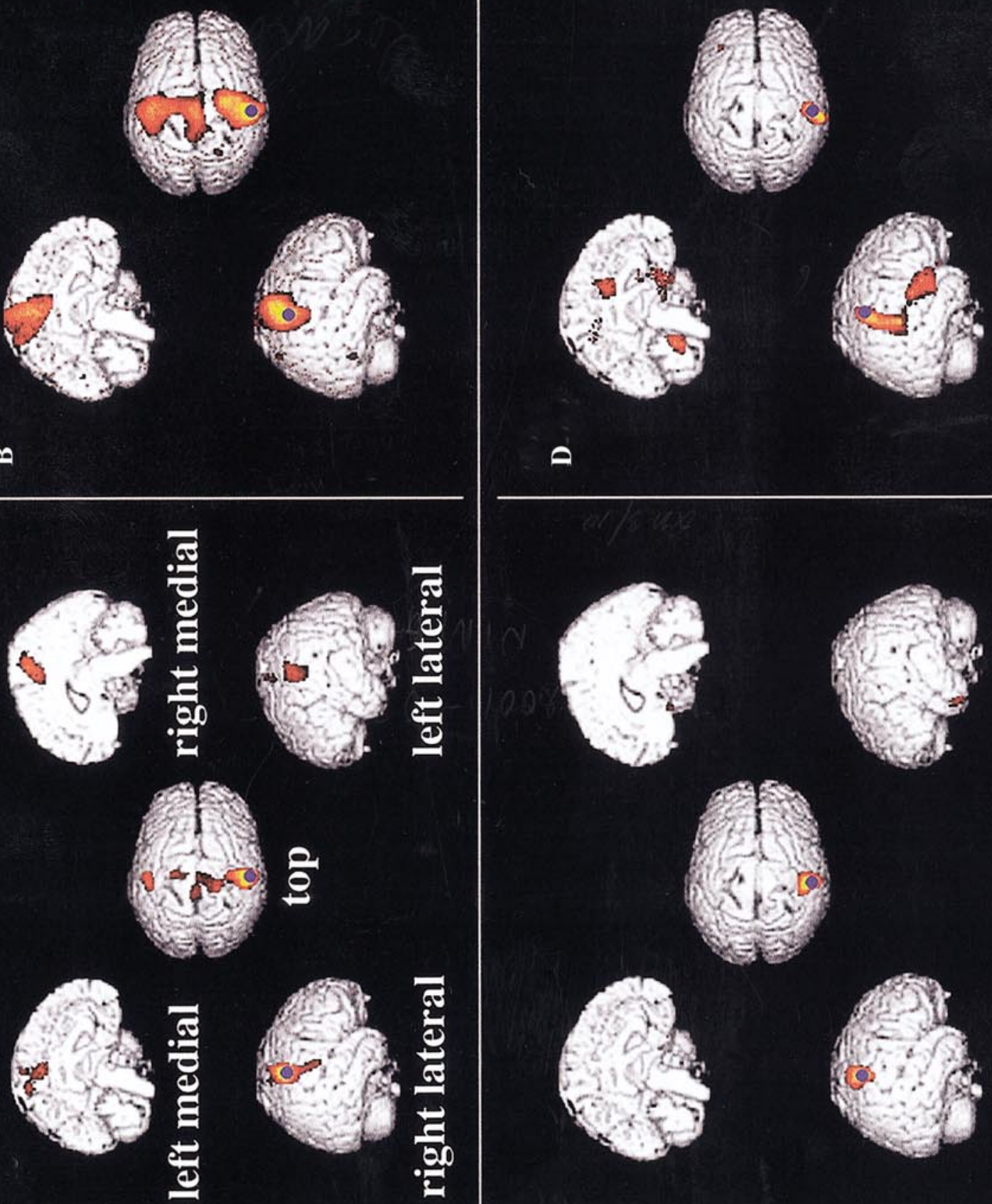

ชं তं

है

$\sum_{0}^{1} \frac{1}{0}$

훈

$\frac{\overline{0}}{0} \frac{\overline{0}}{\overline{0}}$

बह

त

드은은

ठ․

힝

पू

흔휴

อิำ

을형

든 붕

Uิ․ㅡㄹ 은

으뭉

긍

产 ๘

ช 离

安需焉

ช

일

흐으응

ס̄

究袑

幽步

풍 훙

专

ठ웡

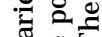

ठㅇ.

주웡

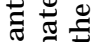

政

훈 흥

的产求

证

ज证
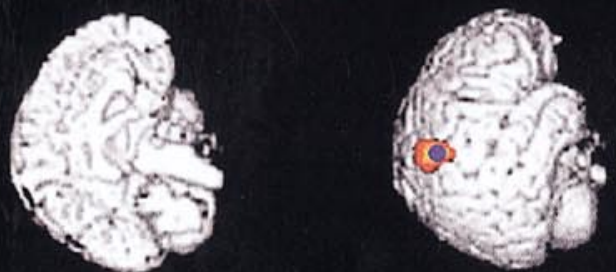

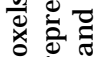

$>$

-i d

نं क्ष $\frac{1}{0}$

는 

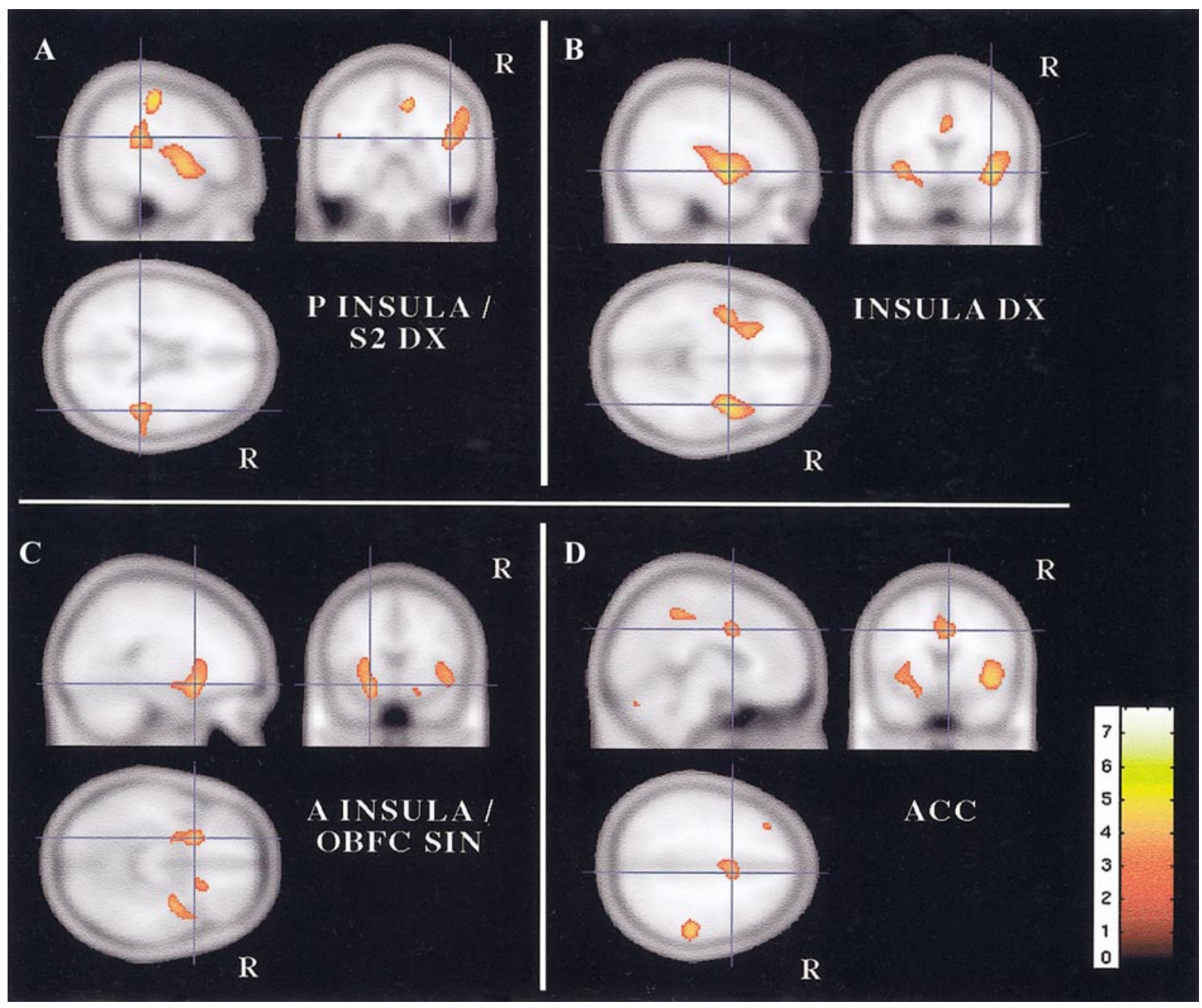

FIG. 2. Voxels in the contralateral S2/posterior insula (A), right and left mid-/anterior insula (B), right and left orbitofrontal cortex bilaterally (C), and the ACC (D), which significantly covaried with the S1-ROI during pb. R indicates the right side of the coronal and horizontal view of the template. The blue cross represents each of the described regions. The sagittal view is from the right hemisphere of the template in (A), (B) and (D), and from the left hemisphere in (C).

When we tested for difference in the regression coefficients during the second minute, the activity of several regions in the primary somatosensory cortex showed a significantly stronger covariation with the S1-ROI activity in the cold condition compared to the pain condition (cb - pb; Table 1). This is similar to the results related to the first minute of pain stimulation. During the second minute of the painful stimulation the activity in the contralateral brainstem, and the mid/anterior insula and the orbitofrontal cortex bilaterally covaried significantly stronger with the S1-ROI activity compared to the second minute of the cold stimulation ( $\mathrm{pb}-\mathrm{cb}$; Table 1).

\section{DISCUSSION}

Although several imaging studies have failed to find any increased activity in the somatosensory regions during pain, it is recognized by most authors that $\mathrm{S} 1$ has a role in pain processing. The important question is whether there are specific noxious stimuli or contexts in which this region is not involved in pain processing, and whether a lack of activity in imaging studies represents such situations. In this regression analysis of a data set where no increased activity was observed, this question was specifically tested. We observed significantly different patterns of covariation with the somatotopic S1 region contralateral to the stimulated hand during the pain conditions compared with the nonpainful control conditions. Although this study cannot distinguish the cause of the lack of activation in S1 (discussed in the introduction) the changed pattern of covariation, suggest a different involvement of S1 during pain as compared with cold processing. Thus, this observation supports our hypothesis that the S1 cortex participated in the processing of pain even though pain 
stimulation as such did not evoke an increase of the S1 activity compared to the control condition.

The variability in an imaging study can be attributed to different sources, e.g., within condition variability and between condition variability. The contrast analysis represents the between condition variance and showed no changes in the S1. Thus, the observed results relay on intra-condition specific rCBF variability. This variability may be attributed to small variations in arousal, cognitive interaction and stimulation input during the noxious stimulation (Petrovic et al., 2000a; Bushnell et al., 1999; Peyron et al., 2000).

The results of this study indicate that there was a significant covariation between the activity of the primary somatosensory regions of the hands during the nonpainful control conditions. This finding is in line with a recent $\mathrm{fMRI}$ study showing that the activity of bilateral homologous regions covaries during rest (Lowe et al., 1998). However, we also observed significant covariations between the S1-ROI and other parts of the postcentral cortex. In fact, all the representation in the S1 from the hand to the foot areas seemed to be functionally connected with the S1-ROI during the second minute of the control condition. This finding may be in accord with another study of cold stimuli that showed increased activation in vast areas of the postcentral cortex (Craig et al., 2000). It is known that there is only a minimum of callosal connections be tween the bilateral hand regions of the S1 (Nieuwenhuys et al., 1988). Thus, it is possible that other neural pathways than the corpus callosum, for example, thalamically relayed pathways, are important for the observed covarying activity in the postcentral gyri. The activity may also represent similar somatosensory inputs from large parts of the body surface which are dependent of factors influencing the whole body, such as temperature and autonomic states (Cechetto and Saper, 1990).

During pain the S1-ROI covaried with a different set of regions that are a part of the pain matrix (including the ACC, the S2/insula, the orbitofrontal cortex). Although many more areas were regressed with the S1ROI during pb than pa, similar but subsignificant regressions were observed in pa and the only significant difference between the regressions during the two pain conditions was observed in the right midanterior insula. The increase in pain intensity/unpleasantness usually takes about $40 \mathrm{~s}$ before the pain rating stabilise (Rainville et al., 1992), which suggest that the perception of pain was possibly more expressed in the pb condition, corroborating a more intense processing between the regions included in the pain network.

Perceptual processing and cognitive functions of the brain seem to be divided by widespread networks of interacting regions, which work cooperatively both se rially and in parallel (Mesulam, 1998). In fact, it seems possible that a given region may be part of different functional networks implementing different types of perceptions or cognitive processing. For example, noxious stimulation is processed by a network consisting of several distinct but widespread regions including the thalamus, ACC, insula, S2 and S1 (Vogt et al., 1993; Willis, 1995). These regions may be involved in different aspects of the pain perception. ACC and insula seem to process the emotional-motivational aspects, while the S1 and S2 seem to process the sensorydiscriminative aspects of pain. Thus, these regions may all work in a coordinated fashion to represent the multidimensional perception of pain in ways analogous to the functional networks for processing visual information, auditory perceptual processing as well as different cognitive functions. However, some of these regions, including S1, are also involved in the processing of nonpainful somatosensory information, suggesting a participation in different networks during different conditions. The presented data are in line with this suggestion since the somatotopic S1 region for the stimulated hand changes its covariational pattern when the perceptions change between the different conditions. For example, the attenuation of the covariation between the S1-ROI and other regions in the post-central gyrus during painful stimulation may indicate that it has a decreased role in the network subserving the somatosensory processing during the nonpainful control condition. Moreover, the significant covariation with other regions involved in pain processing observed during the second minute of painful stimulation, indicates that the S1 indeed may change its pattern of functional relations. I mportantly, this functional relation was significantly stronger for the insula and the orbitofrontal cortex during the second minute of pain processing compared to the second minute of the control condition. Thus, the somatotopic S1 area for the stimulated hand will work more in concert with regions important for the processing of pain during the noxious stimulation as compared to the nonpainful cold stimulation.

It is also interesting that the observed regions, which covaried with theS1-ROI are similar to those that have been found to correlated with pain ratings (for example see Coghill et al., 1999). Thus, both methods support the hypothesis that a pain network work in a parallel and distributed fashion during pain processing.

However, it should be noted that in an regression analysis, the observation of a significant functional relation does not necessarily imply a causal relation but may alternatively be the result of a common source of influence, e.g., the same noxious input. In this case, it would anyway indicate that activity in S1 is related to pain processing although there is no change in differential activity between conditions. For example, the strong covariation between the S1-ROI and the insula may indicate a similar source of noxious input to those structures. Both BAЗa and the insula seem to have 
input from the VMpo thalamic nucleus, and this network has previously been hypothesised to represent the physiological status of the body tissues based by noxious and temperature input to the brain (Craig et al., 1999, 2000).

In conclusion, the study shows that a regression approach may disclose involvement of a region in pain processing, which is not evident in a standard subtraction analysis approach. More specifically, this study suggests that the primary somatosensory cortex may be involved in pain processing although no net-increase was observed in the subtraction analysis. Thus, any suggestion that $\mathrm{S} 1$ is not involved in pain processing during specific conditions or stimulations where there is a lack of S1 activity stand to be reinterpreted. Also, this study supports the hypothesis that S1 may be a part of different networks during noxious cold as compare with nonpainful cold stimulation.

\section{ACKNOWLEDG MENTS}

Grants from the Swedish Medical Research Council (8276), Förenade Liv, the Karolinska Institute, the Swedish Medical Association, the Knut and Alice Wallenberg foundation, and the Family Hedlund Foundation supported this work. The authors are grateful to all participating from the PET facility.

\section{REFERENCES}

Berridge, M. S., Cassidy, E. H., and Terris, A. H. 1990. A routine, automated synthesis of oxygen-15-labeled butanol for positron tomography. J . Nucl. Med. 31: 1727-1731.

Bushnell, M. C., Duncan, G. H., Hofbauer, R. K., Ha, B., Chen, J . I., and Carrier, B. 1999. Pain perception: Is there a role for primary somatosensory cortex? Proc. Natl. Acad. Sci. USA 96: 7705-7709.

Casey, K. L., Minoshima, S., Morrow, T. J ., and Koeppe, R. A. 1996. Comparison of human cerebral activation patterns during cutaneous warmth, heat pain and deep cold pain. J . Neurophysiol. 76: 571-581.

Casey, K. L., Morrow, T. J ., Lorenz, J ., and Minoshima, S. 2001. Temporal and spatial dynamics of human forebrain activity during heat pain: Analysis by positron emission tomography, J . Neurophysiol. 85: 951-959.

Cechetto, D. F., and Saper, C. B. 1990. The role of the cerebral cortex in autonomic function. In Central Regulation of Autonomic Functions (A. D. Loewy and K. M. Spyer, Eds.), pp. 208-223. Oxford Univ. Press, New York.

Coghill, R. C., Sang, C. N., Maisog, J . M., and Iadarola, M. J . 1999. Pain intensity processing within the human brain: a bilateral, distributed mechanism. J . Neurophysiol. 82: 1934-1943.

Craig, A. D., Chen, K., Bandy, D., and Reiman, E. M. 2000. Thermosensory activation of insular cortex. Nature Neurosci. 3: 184190.

Craig, A. D., and Dostrvsky, J. O. 1999. Medulla to thalamus. In Textbook of Pain (P. D. Wall and R. Melzack, Eds.), pp. 183-214. Churchill Livingstone.

Craig, A. D., Reiman, E. M., Evans, A., and Bushnell, M. C. 1996. Functional imaging of an illusion of pain. Nature 384: 258-260.

Derbyshire, S. W., and J ones, A. K. 1998. Cerebral responses to a continual tonic pain stimulus measured using positron emission tomography. Pain 76: 127-135.
Di Piero, V., Ferracuti, S., Sabatini, U., Pantano, P., Cruccu, G., and Lenzi, G. L. 1994. A cerebral blood flow study on tonic pain activation in man. Pain 56: 167-173.

Di Piero, V., Fiacco, F., Tombari, D., and Pantano, P. 1997. Tonic pain: A SPET study in normal subjects and cluster headache patients. Pain 70: 185-189.

Friston, K. 1994. Functional and effective connectivity: A synthesis. Hum. Brain Mapp. 2: 56-78.

Friston, K. J., Holmes, A. P., Worsley, K. J., Poline, J .-P., and Frackowiak, R. S. J . 1995. Statistical parametric maps in functional imaging: A general linear approach. Hum. Brain Mapp. 2: 189-210.

Friston, K. J ., Buechel, C., Fink, G. R., Morris, J., Rolls, E., and Dolan, R. J. 1997. Psychophysiological and modulatory interactions in neuroimaging. Neurol mage 6: 218-229.

Greenspan, J. D., and Winfield, J. A. 1992. Reversible pain and tactile deficits associated with a cerebral tumor compressing the posterior insula and parietal operculum. Pain 50: 29-39.

Greitz, T., Bohm, C., Holte, S., and Eriksson, L. 1991. A computerized brain atlas: Construction, anatomical content and some applications, J . Comput. Assist. Tomogr. 15: 26-38.

Head, H., and Holmes, G. 1911. Sensory disturbances from cerebral lesions. Brain 34: 102-254.

Ingvar, M. 1999. Pain and functional imaging. Philos. Trans. R. Soc. London, Series B: Biol. Sci. 354: 1347-1358.

Ingvar, M., Eriksson, L., Greitz, T., Stone-Elander, S., Dahlbom, M., Rosenqvist, G., af Trampe, P., and von Euler, C. V. 1994. Methodological aspects of brain activation studies: Cerebral blood flow determined with $\left[{ }^{15} \mathrm{O}\right]$ butanol and positron emission tomography. J . Cereb. Blood Flow Metab. 14: 628-638.

Kenshalo, D. R., J r., Chudler, E. H., Anton, F., and Dubner, R. 1988. SI nociceptive neurons participate in the encoding process by which monkeys perceive the intensity of noxious thermal stimulation. Brain Res. 454: 378-382.

Kenshalo, D. R. J ., and I sensee, O. 1983. Responses of primate S1 cortical neurons to noxious stimuli. J. Neurophysiol. 50: 14791496.

Kenshalo, D. R. J ., Thomas, D. A., and Dubner, R. 1991. Primary somatosensory cortical lesion reduce the monkeys' ability to discriminate and detect noxious thermal stimulation. Soc. Neurosci. Abstr. 17: 1206.

Lowe, M. J., Mock, B. J ., and Sorenson, J. A. 1998. Functional connectivity in single and multislice echoplanar imaging using resting-state fluctuations. Neuroimage 7: 119-132.

Mesulam, M.-M. 1998. From sensation to cognition. Brain 121: 1013-1052.

Nieuwenhuys, R., Voogd, J ., and van Huijzen, C. 1988. Long association and commissural connections. In The Human Central Nervous System, pp. 365-375. Springer-Verlag.

Petrovic, P., Ghatan, P. H., Peterson, K.-M., Stone-Elander, S., and Ingvar, M. 2000a. Pain related cerebral activation is altered by a distracting cognitiv task. Pain 85: 19-30.

Petrovic, P., Peterson, K.-M., Hansson, P., and Ingvar, M., $2000 \mathrm{~b}$. Habituation to pain-rCBF and autonomic effects of stimulus duration during cold pressor test. Neurol mage 11: S717.

Peyron, R., Garcia-Larrea, L., Gregoire, M. C., Costes, N., Convers, P., Lavenne, F., Mauguiere, F., Michel, D., and Laurent, B. 1999. Haemodynamic brain responses to acute pain in humans: sensory and attentional networks. Brain 122: 1765-1780.

Peyron, R., Laurent, B., Garc, and a-Larrea, L. 2000. Functional imaging of brain responses to pain. A review and meta-analysis. Neurophysiol. Clin. 30: 263-288. 
Rainville, P., Feine, J . S., Bushnell, M. C., and Duncan, G. H. 1992. A psychophysical comparison of sensory and affective responses to four modalities of experimental pain. Somatosensory Motor Res. 9: 265-277.

Robinson, C. J ., and Burton, H. 1980. Somatic submodality distribution within the second somatosensory (S2), 7b, retroinsular, postauditory, and granular insular cortical areas of M. fascicularis. J . Comp. Neurol. 192: 93-108.

Tommerdahl, M., Delemos, K. A., Vierck, C. J ., J r., Favorov, O. V., and Whitsel, B. L. 1996. Anterior parietal cortical response to tactile and skin-heating stimuli applied to the same skin site. J. Neurophysiol. 75: 2662-2670.
Treede, R. D., Kenshalo, D. R., Gracely, R. H., and J ones, A. K. 1999. The cortical representation of pain. Pain 79: 105-111.

Vogt, B. A., Sikes, R. W., and Vogt, L. J . 1993. Anterior cingulate cortex and the medial pain system. In Neurobiology of Cingulate Cortex and Limbic Thalamus: A Comprehensive Handbook (B. A. Vogt and M. Gabriel, Eds.), pp. 313-344. Birkhäuser, Boston, MA.

Wienhard, K., Dahlbom, M., Eriksson, L., Michel, C., Bruckbauer, T., Pietrzyk, U., and Heiss, W. D. 1994. The ECAT EXACT HR: Performance of a new high resolution positron scanner.J . Comput. Assist. Tomogr. 18: 110-118.

Willis, W. D. J . 1995. Cold, pain and the brain. Nature 373: 19-20. 